\title{
Reverberation Mapping and Broad-Line Region Models
}

\author{
Dan Maoz \\ School of Physics and Astronomy and Wise Observatory, Tel-Aviv \\ University, Tel-Aviv 69978, Israel
}

\begin{abstract}
I review what we have learned about the BLR from reverberation mapping, point to some problems and complications that have emerged, and outline some future directions.
\end{abstract}

\section{Basics}

Reverberation mapping is a technique in which variability data exhibiting light travel-time effects are used to to derive information on the geometry and kinematics of a source. Reviews of the basic principles and methods of reverberation mapping as applied to AGNs have appeared in Peterson $(1988 ; 1993)$ and in Netzer (1990). More detailed information is compiled in the proceedings of a workshop dedicated to the topic (Gondhalekar, Horne, \& Peterson 1994).

The fundamental equation of reverberation mapping is the convolution equation relating the emission-line and continuum light curves, $L(t)$ and $C(t)$ :

$$
L(t)=\int \Psi(\tau) C(t-\tau) d \tau
$$

The kernel of the convolution is the transfer function, $\Psi(\tau)$, which contains the geometrical and physical information inherent to the reprocessing of energy that relates the two light curves. It is the 'Green's function' of the system, or the hypothetical response of a BLR emission line to a very brief continuum burst. An analogous two-dimensional transfer function, $\Psi(\tau, v)$ relates every projected-velocity bin, $v$, in the line profile to the continuum light curve (see the contribution by $K$. Horne in this volume). $\Psi$ provides a 'picture' of the BLR in time-delay (or time-delay/projected-velocity) space. With some assumptions of symmetry, one can then guess the full six-dimensional geometry and kinematics of the BLR.

In the next sections I will sketch my view of the current status of the field, including how well the basic assumptions of reverberation mapping seem to be faring, and what we have learned to date about the BLR. I will outline the directions I think echo mapping will take in the near future.

\section{Problems}

With the execution of increasingly ambitious reverberation-mapping campaigns (see this volume) and improved data, we have seen a basic prediction of the 
NGC 5548

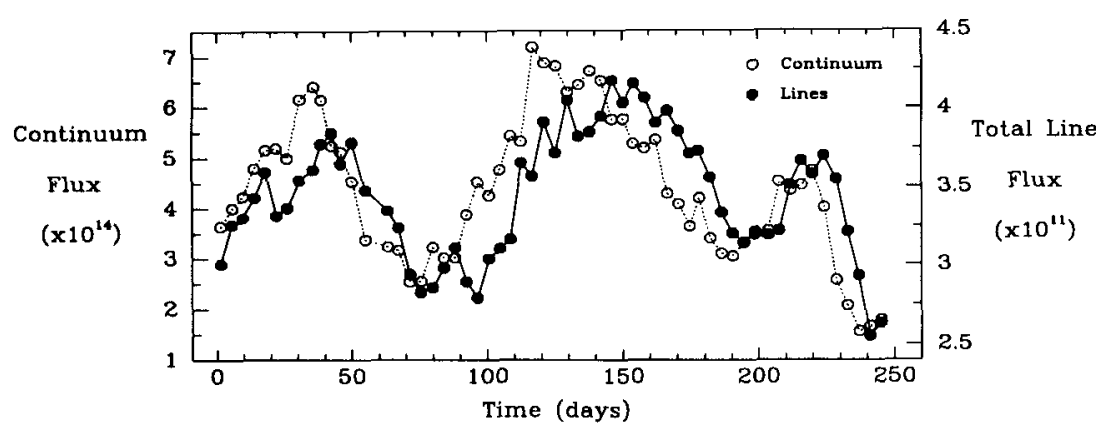

Figure 1. UV $(1350 \AA)$ continuum light curve (empty circles, left vertical scale, in $\operatorname{erg~s}^{-1} \mathrm{~cm}^{-2} \AA^{-1}$ ) for NGC 5548, and total observed emission-line flux (filled circles, right vertical scale, in $\operatorname{erg~s}^{-1} \mathrm{~cm}^{-2}$ ), during the 1989 IUE/optical monitoring campaign.

AGN photoionization model beautifully confirmed, namely, that emission-line light curves mimic the continuum behavior, but with a lag due to light-travel time effects. Among the most illuminating results are those from the joint IUE/optical campaign on NGC 5548 in 1989 (Clavel et al. 1991; Peterson et al. 1991; Dietrich et al. 1993; Maoz et al. 1993). The gross structure of the light curves for the strong lines, whose sum is shown and compared to the continuum in Fig. 1, is just that expected.

However, the NGC 5548 data also revealed various peculiarities, some of which point to inadequacies in the basic assumptions of reverberation mapping. Evidence for such effects has been seen in other objects as well. The three main complications which seem to be indicated by variability data are non-linear response of the emission lines, 'misrepresentation' of the ionizing continuum by the observed optical or UV continuum, and significant structural evolution of the BLR on time scales of a year.

The nonlinear behavior is most clearly visible in the NGC 55481989 light curve of $\operatorname{CIV} \lambda 1549$. The total energy in the third continuum 'event' is much less than that of the previous two events and, if the line light curve were a linear convolution of the continuum light curve, would produce a correspondingly weak feature in the emission-line light curve. Instead, the CIV flux rises to the same amplitude it had in response to the previous two events. In retrospect, evidence for nonlinearities in BLR response has been around for a long time. Wamsteker \& Colina (1986) first pointed out the saturation in the C IV $\lambda 1549$ level with increasing continuum flux in Fairall 9. A more moderate manifestation of the same effect, whereby C IV responds less than Ly $\alpha$ to continuum changes, can be seen, e.g., in O'Brien et al. (1996) for the much smaller continuum variations of NGC 5548 in 1989. In all AGNs, the fractional amplitude of the emission-line variations is always considerably smaller than that of the continuum variations, once known constant components such as narrow lines and galaxy light have 

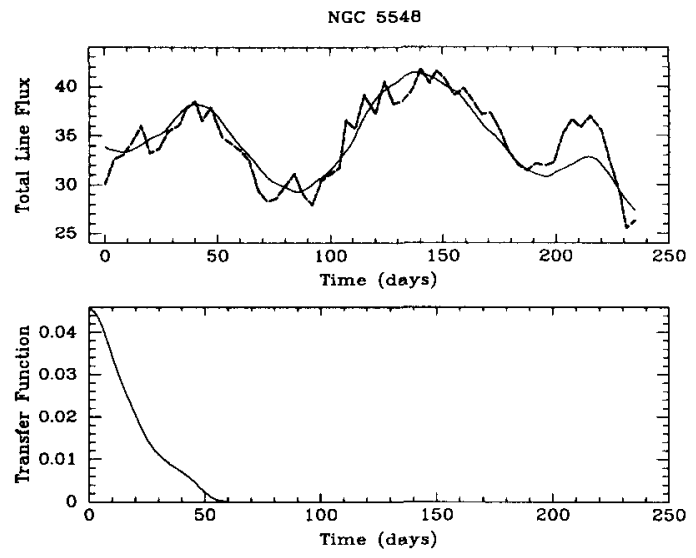

Figure 2. Top panel: Jagged dashed line is the total emission line light curve of NGC 5548, same as in Fig. 1. Bottom panel: Transfer function obtained by maximum entropy inversion of the total light curve with the UV continuum light curve, excluding the third 'event'. The smooth solid line in the top panel is the reconstructed emission line light curve obtained by convolving the continuum light curve with the transfer function in the bottom panel. The third event is poorly reproduced for any such monotonically decreasing transfer function.

been accounted for. Clearly, some of the gas contributing to the broad-line flux does not respond to the observed continuum variations.

To a certain extent, non-linearities in the response of individual lines are predicted by conventional photoionization models (e.g. Goad, O'Brien, \& Gondhalekar 1993). However, the details of the response of a particular line cannot be the entire story behind the problem, because as long as each BLR cloud remains optically thick to the ionizing radiation, the energy from each ionizing photon must come out as one line photon or another (or several). Therefore, the nonlinear aspects should disappear in the total emission-line light curve. (This is not a new idea; see Blandford \& McKee [1982], §II.a.) Returning to Fig.1, the third-event problem is, indeed, considerably reduced in the total line light curve. However, the problem has not disappeared.

Figure 2 (top panel) shows the same total line curve (now shown as a jagged dashed line). Superposed on it is a reconstructed light curve (smooth solid line) obtained with a maximum entropy inversion that was forced to produce a transfer function that is monotonically decreasing (bottom panel). Maximum entropy methods applied to the NGC 5548 data have generally produced separate aliasing peaks in the transfer function which 'conspire' with previous continuum events in order to ameliorate the third-event problem. Figure 2 shows that if such aliasing is not allowed, the amplitude of the third event is still much too high, even in the total line flux.

Possible explanations are either that there is a fraction of optically thin gas in the BLR (Shields, Ferland, \& Peterson 1995; O'Brien et al. 1996); or 
the continuum behavior we see is not the ionizing continuum behavior that the BLR gas sees. This can come about if the continuum emission is not emitted isotropically, or if the ionizing continuum is not strictly linearly proportional to the observed continuum longward of the Lyman edge (as proposed, e.g., by Clavel \& Santos-Lleó 1990, to explain the CIV saturation in Fairall 9).

There is already evidence for the latter possibility in NGC 5548, in the well-established hardening of the continuum as it rises (e.g. Maoz et al. 1993). Additional evidence for continuum 'misbehavior' comes from the intensive 1993 multiwavelength campaign on NGC 4151 (Crenshaw et al. 1996; Kaspi et al. 1996a; Warwick et al. 1996; Edelson et al. 1996). Figure 9 of Kaspi et al. (1996a) shows a scaled version of the IUE $2700 \AA$ continuum light curve superposed on the $1275 \AA$ continuum light curve. The two light curves, just a factor of 2 apart in energy, are clearly not just linearly scaled versions of each other. Things might be just as bad or worse shortward of $912 \AA$. Additional evidence for problems with the 'surrogate' continuum is that the optical continuum variation amplitude is similar to that in a previous campaign on the same object (Maoz et al. 1991) but the Balmer-line variations are much smaller in the more recent campaign. If an imperfect correlation between the observed and ionizing continua is the source of the problems mentioned above, it could a pose a difficult hurdle, as the ionizing continuum itself cannot be directly observed.

A third complication is the detection of a time-variable lag in several objects (Netzer \& Maoz 1990; Peterson et al. 1994; Wanders \& Horne 1994), suggesting a BLR that evolves on time scales of about one year. Possibly related is the fact that discrete velocity components are known to appear and disappear in AGN line profiles, in a manner unrelated to the continuum variations (Wanders \& Peterson 1996). Wanders (this volume) has suggested that these components are fluctuations in the fraction of orbiting BLR clouds that are illuminated by an ionization cone, rather than real changes in the BLR geometry. In any case, the observed evolution in the BLR provides an exciting new dimension in reverberation mapping, but may mean that long, sparsely sampled observations are not an alternative to intensive, season-long campaigns.

Finally, there may have been some over interpretation of the data. It has become common to take variability data and to attempt to invert the convolution equation directly to recover $\Psi$ (e.g., Maoz et al. 1991; Krolik et al. 1991; Horne, Welsh \& Peterson 1991; Peterson et al. 1994; Wanders \& Horne 1994). However, even the best currently available variability data are very noisy, and therefore may produce non-unique transfer functions when deconvolved. As an example, let us look at the $\mathrm{H} \beta$ data for NGC 5548. Horne et al. (1991) and Peterson et al. (1994) have found that the maximum-entropy derived transfer function is peaked at 20 days, and has low-amplitude at zero lag. They interpreted this as meaning that there is little variable $\mathrm{H} \beta$ line-emission coming from our line of sight to the nucleus, either because of the BLR geometry or due to optical-depth effects in the line.

In Figure 3, 1 have taken the five-year-long optical continuum light curve of NGC 5548 (Peterson et al. 1994; Korista et al. 1995), linearly interpolated it to one-day intervals, and convolved it with three different transfer functions: a delta-function peaked at 20 days, a top-hat function that is positive from 0 to 40 days, and a triangular function peaked at 0 days and decreasing to zero at 60 


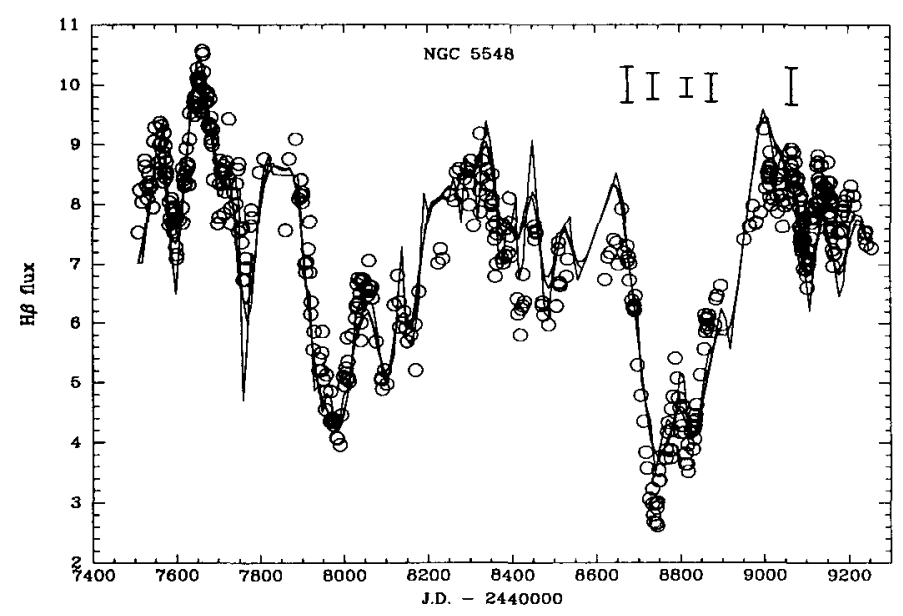

Figure 3. NGC 5548 5-year $\mathrm{H} \beta$ light curve (empty circles). Some typical error bars are shown in upper right corner. The three solid lines are the convolution of the interpolated optical continuum light curves with three different-shaped transfer functions: a delta-function, a top-hat, and a triangle peaked at zero lag. Can the data distinguish between these models?

days. In my talk, I argued that the differences between the light curves produced by these very different-shaped transfer functions are minute compared to the uncertainties in the line measurements themselves, and that these data cannot distinguish between these transfer functions, and in particular between transfer functions peaked at zero-lag and away from zero. After my talk, Keith Horne challenged me to add simulated measuring errors to the fake light curves, and send them to him, which I did. To my surprise, Keith's MEMECHO program recovered the transfer functions well enough for him to easily guess by a process of elimination the correct transfer function to assign to each light curve. On the other hand, Wanders \& Peterson (1996) have recently concluded from analysis of a newly-reduced version of the same observations that the NGC $5548 \mathrm{H} \beta$ transfer function cannot yet be uniquely determined.

\section{What Have We Learned?}

Despite the problems outlined above, we have learned quite a bit about the BLR from reverberation mapping. An important result from the 1989 NGC 5548 campaign, supported by the $1993 I U E+H S T$ campaign of the same object (Korista et al. 1995), is that the BLR is stratified in ionization. Different emission lines respond to the continuum with different lags. The range in lags spans a factor of 5 , perhaps more, and there is a trend for the most highly ionized species to have the smallest lags (i.e., be emitted at preferentially small radii). This result has outmoded the single-cloud photoionization models that were common for 
many years. It is now clear that BLR gas exists at a range of radii. Baldwin et al. (1995; see also Baldwin, this volume) have argued that such stratification is a natural consequence of a 'thick' BLR, with different lines reaching their peak emission efficiencies at different radii.

The question of BLR geometry and kinematics is still unresolved. I note that the data on the best-studied object, NGC 5548, have produced papers advocating a variety of models. Wanders et al. (1995) argue for a spherical BLR with randomly inclined circular orbits, illuminated by an ionizing bi-cone viewed approximately end-on. (This geometry is probably indistinguishable from one with random radial orbits, with the bi-cone approximately in the plane of the sky.) Done \& Krolik (1996) find the data consistent with a thick spherical geometry with Keplerian orbits. Chiang \& Murray (1996; see also Murray, this volume) and Rokaki (this volume) model the results with a Keplerian disk. The data cannot yet distinguish between these models. We probably can say that the BLR transfer function is resolved on time scales of days, and therefore has some structure. I hope that future experiments will see the details of this structure.

We do know some things that the BLR is not. Models invoking pure radial flow (whether infall or outflow) as the dominant line broadening mechanism have been long discussed. A prediction of any such model is a lag between the blue and red wings of the profile, comparable to the lag of the total line flux behind the continuum. Every experiment that has tested this prediction with adequate temporal resolution has obtained a null result (e.g., Maoz et al. 1991, Wanders et al. 1995). It will be interesting to see if these null results hold up in higher-luminosity objects; radial flows are utilized to explain the line shifts observed in quasars (see Espey, this volume).

\section{The BLR at Higher Luminosities}

Until recently, quasars have been largely neglected by echo mappers. This has been due to their faintness (and hence inaccessibility to IUE and to small telescopes), their frequent lack of narrow emission lines (still the most popular flux calibrator), and some prejudices about their (presumably long and nonpaper/thesis-producing) variation and response time scales. The small amount of data that did exist on quasar emission-line variability (often only two or three epochs per object) produced controversial and sometimes contradicting interpretations (see Peterson 1993, for a review).

Happily, reverberation results for higher-luminosity AGNs have begun to appear. Carone et al. (1996) measured a surprisingly large $\mathrm{H} \beta /$ continuum lag (100 days) in Mrk 509, a luminous Seyfert galaxy. For the past five years, a collaboration between Wise and Steward Observatories has been monitoring 28 bona-fide quasars from the PG sample (Maoz et al. 1994; Kaspi et al. 1996b; see Kaspi, this volume). This program has demonstrated that reverberation mapping indeed works in quasars, much as it does in Seyferts. The first clear lags that have been measured, in two quasars, are also of about 100 days.

Does the BLR size scale with luminosity as $R_{B L R} \propto L^{1 / 2}$, as long predicted based on the overall great similarity of AGN spectra over many orders of magnitude in luminosity? Figure 3 in Kaspi's contribution (this volume) shows the BLR size as a function of luminosity. The two highest luminosity points are 
the two new quasar measurements. While more quasar BLR radii (which are upcoming from the Wise-Steward program) are desirable, the trend in the figure is certainly suggestive of the expected relation.

\section{The Future}

With the demise of IUE (after an incredibly long and fruitful service) a rethinking of strategy is required of echo mappers. To continue progress in understanding the BLR of Seyferts by this technique, a significant improvement is required in the sampling frequency (approximately daily sampling lasting about 6 months) and in the number of AGNs that are monitored. High spectral resolution and $S / N$ may also start producing the long-sought 2-D transfer functions. Measurement errors should remain below the $2-3 \%$ level, since we now know that relatively low-amplitude variations (tens of percent) are the norm in non-blazar AGNs. All these constraints point to the need for one or more dedicated AGNmonitoring ground-based telescopes. High efficiencies could be obtained with $2-3 \mathrm{~m}$ class apertures, especially if a high degree of automation is implemented. Two telescopes can minimize to a large extent the gaps due to weather.

Progress with quasars may be easier. Going down to $B=20 \mathrm{mag}$, there are 15 quasars per square degree. Using multi-object spectrographs with wide fields, such as are available on many large telescopes, one can monitor tens of quasars simultaneously. The slower response time (further dilated by $1+z$ in high- $z$ objects) also means that the sampling constraint can be relaxed. With once-aweek observations on a $4 \mathrm{~m}$ telescope over several years, the BLRs of hundreds of quasars, spanning both luminosity and look-back time, can be studied.

\section{Conclusions}

The work of the past decade has demonstrated that reverberation mapping works in both low and high-luminosity AGNs, and has huge potential. While we cannot yet say that we have determined the structure or kinematics of any BLR, we are at the point where we can discern time-resolved transfer functions in the best-studied objects. Improved data will reveal the details of the transfer functions, and perhaps of the BLRs themselves. We have already learned about ionization stratification, and the inapplicability of some models, e.g., pure radial flow models for Seyferts.

The steadily improving quality of the observations have also revealed complications, indicative of the approximations behind some of the basic assumptions: non-linear response, suggestive of the presence of some optically thin gas, and BLR evolution, vs. the usually assumed stationarity. These are 'good' complications, in that they point us to a more realistic picture of the BLR. A more worrisome complication is the possibility of a 'misbehaving' observed continuum that does not completely represent the ionizing continuum seen by the BLR. Future, efficient, reverberation surveys, such as I have outlined, could show the degree to which such continuum misbehavior is a problem. 


\section{References}

Baldwin, J., Ferland, G., Korista, K., \& Verner, D. 1995, ApJ, 455, L119.

Blandford, R.D. \& McKee, C.F. 1982, ApJ, 255, 419.

Carone, T.E., et al. 1996, ApJ, in press.

Chiang, J., \& Murray, N. 1996, ApJ, in press.

Clavel, J., et al. 1991, ApJ, 366, 64.

Clavel, J., \& Santos-Lleó, M. 1990, A\&A, 230, 3.

Crenshaw, D. M., et al. 1996, ApJ, 470, in press.

Dietrich, M., et al. 1993, ApJ, 408, 416.

Done, C., \& Krolik, J. H. 1996, ApJ, in press.

Edelson, R. A., et al. 1996, ApJ, 470, in press.

Goad, M.R., O'Brien, P. T., \& Gondhalekar, P. M. 1993, MNRAS, 263, 149.

Gondhalekar, P. M, Horne, K., \& Peterson, B. M. 1994, Reverberation Mapping of the Broad-Line Region in Active Galactic Nuclei (San Francisco: ASP).

Horne, K., Welsh, W.F., \& Peterson, B. M. 1991, ApJ, 367, L5.

Kaspi, S., et al. 1996a, ApJ, 470, in press.

Kaspi, S., Smith, P. S., Maoz, D., Netzer, H., \& Jannuzi, B. T. 1996b, ApJL, in press.

Korista, K. T., et al. 1995, ApJS, 97, 285.

Krolik, J. H., Horne, K., Kallman, T. R., Malkan, M. A., Edelson, R. A., \& Kriss, G. A. 1991, ApJ, 371, 541.

Maoz, D., Netzer, H., Leibowitz, E., Brosch, N., Laor, A., Mendelson, H., Beck, S., Almoznino, E., \& Mazeh, T. 1991, ApJ, 367, 493.

Maoz, D., et al. 1993, ApJ, 404, 576.

Maoz, D., Smith, P. S., Jannuzi, B. T., Kaspi, S., \& Netzer, H. 1994, ApJ, 421, 34 .

Netzer, H. 1990, in Active Galactic Nuclei, ed. T.J.-L. Courvoisier and M. Mayor, Saas-Fee Course 20 (Berlin: Springer-Verlag).

Netzer, H., \& Maoz, D. 1990, ApJ, 365, L5.

O'Brien, P. T., Goad, M. R., \& Gondhalekar, P. M. 1996, MNRAS, in press.

Peterson, B. M., 1988, PASP, 100, 18.

Peterson, B. M., et al. 1991, ApJ, 368, 119.

Peterson, B. M. 1993, PASP, 105, 247.

Peterson, B. M., et al. 1994, ApJ, 425, 622.

Shields, J. C., Ferland, G. J., \& Peterson, B. M. 1995, ApJ, 441, 507.

Wamsteker, W., \& Colina, L. 1986, ApJ, 311, 617.

Wanders, I., et. al. 1993, A\&A, 269, 39.

Wanders, 1., \& Horne, K. 1994, A\&A, 289, 76.

Wanders, I., et al. 1995, ApJ, 453, L87.

Wanders, I., \& Peterson, B. M. 1996, ApJ, 466, 174.

Warwick, R., et al. 1996, ApJ, 470, in press. 